

\title{
LOW ENERGY BEAM-GAS SPECTROSCOPY OF HIGHLY IONISED ATOMS
}

\author{
J. Desesquelles, A. Denis, M. Druetta, S. Martin
}

\section{To cite this version:}

J. Desesquelles, A. Denis, M. Druetta, S. Martin. LOW ENERGY BEAM-GAS SPECTROSCOPY OF HIGHLY IONISED ATOMS. Journal de Physique Colloques, 1989, 50 (C1), pp.C1-71-C1-84. 10.1051/jphyscol:1989107 . jpa-00229298

\section{HAL Id: jpa-00229298 https://hal.science/jpa-00229298}

Submitted on 1 Jan 1989

HAL is a multi-disciplinary open access archive for the deposit and dissemination of scientific research documents, whether they are published or not. The documents may come from teaching and research institutions in France or abroad, or from public or private research centers.
L'archive ouverte pluridisciplinaire HAL, est destinée au dépôt et à la diffusion de documents scientifiques de niveau recherche, publiés ou non, émanant des établissements d'enseignement et de recherche français ou étrangers, des laboratoires publics ou privés. 
LOW ENERGY BEAM-GAS SPECTROSCOPY OF HIGHLY YONISED ATOMS

\author{
J. DESESQUELles, A. DENIS, M. DRUETTA and S. MARTIN \\ Laboratoire de Spectrométrie Ionique et Moléculaire, CNRS LA-171, \\ Université Lyon I, Bât. 205, $43 \mathrm{Bd}$ du 11 Novembre 1918, F-69622 \\ Villeurbanne Cedex, France
}

\begin{abstract}
Résumé - Nous présentons la source spectroscopique que constitue un faisceau d'ions multichargés de basse énergie excité par collision dans un gaz et nous la comparons a d'autres sources de lumière. Ses propriétés particulières sont décrites et nous donnons quelques résultats, obtenus par spectroscopie dans le visible et l'u.v., sur la structure d'ions lourds multichargés dans des états simplement excités, doublement excités, de Rydberg et de Rydberg doublement excités.
\end{abstract}

Abstract - Features of low energy beam-gas spectroscopic source are reviewed and compared to those of other light sources. Measurement techniques are surveyed. They include the study of wavelength of heavy multiply charged ions in visible and u.v. ranges from normal excited states, doubly excited states, high $n$ levels and doubly excited Rydberg levels.

\title{
1 - INTRODUCTION
}

Experimental studies of atomic structures are usually performed by applying suitable excitation device to the atoms and determining the energies and intensity of the radiation. In the case of highly ionised atoms, the measured atomic data, besides their intrinsic and applied interest, serve as tests of various approximations used in calculations of atomic structures taking correlation. relativistic and QED contributions into account.

Two techniques are available to produce multiply charged ions into excited states. We can cook the atoms in energetic plasmas or we can accelerate the atoms to high energies and then strip off the electrons by passage through matter.

In beam-foil method, stripping dependence on beam velocity can be evaluated from the Bohr criterion. High charge states need high velocity. Average charge to nuclear charge ratio obeys, at high energies, the formula [1] $\bar{q} / Z=1-\exp \left(-\mathrm{v} Z^{-1 / 3}\right)$ as a function of beam velocity (in atomic units). At $2 \mathrm{MeV}$ Van de Graaff energies such ions as lithiumlike oxygen, neonlike argon and low charge heavy ions have been studied. At $1 \mathrm{MeV} /$ nucleon charge states 15 and 40 are efficiently produced in argon and uranium respectively. At energies of GANIL (20-30 MeV/A) a good break up of the $\mathrm{K}$ shell is obtained in krypton and xenon [2]. Bare uranium ions have been produced on Super Hilac, at Berkeley [3]. Distribution of charge states at equilibrium is narrow ( \pm 2 ) for $Z \leq 10$ but for heavy elements a dozen of charge states may be important.

When the thin solid target is replaced by a gaseous target, produced charge states are lower. 
Photoionisation is the clearest process capable of producing ionised states. Ions have been produced using synchrotron $X$-ray source. In argon, a distribution of charge states peaked at Ar $4+-$ Ar ${ }^{5}$ has been obtained and stored at Brookhaven [4]. Ionisation of ion beams would be interesting.

Ions can also be protuced in a gas bombarded by a primary fast highly charged ion beam. Recoil ions are slow but every charge state contributes. At $1.14 \mathrm{MeV} / \mathrm{A}, \mathrm{U}^{44+}$ produces $\mathrm{Ar}{ }^{10+}, \mathrm{Kr}^{11+}, \mathrm{Xe} 18+$ with cross-sections greater than $10^{-16} \mathrm{~cm}^{2}[5]$.

Highly stripped ions are also present in energetic plasmas, which can be produced in several ways. Charge states are broadly distributed. Plasma and beam-gas interaction have a comparable stripping power when the plasma electron temperature $(\mathrm{eV})$ is 90 times the projectile energy (MeV/ nucleon) [6]. Charges are about the same in a $1 \mathrm{keV}$ plasma than in a $3 \mathrm{MeV} / \mathrm{A}$ foil stripped ion beam.

Here we are interested by a source of multiply charged ions combining several advantages of other sources. Among new ion sources, EBIS and ECRIS are particularly efficient to produce highly ionised atoms. ECR ion sources [7], available for a few years, currently deliver micro amperes of $\mathrm{Ne}^{8+}, \mathrm{Ar}^{10+}, \mathrm{Kr}^{12+}$ at $\mathrm{keV}$ energies. If ions are extracted from the plasma and charge and mass analysed, a given $\mathrm{X}^{q+}$ ion beam, at keV energies, can capture an electron in a gas target and produce excited $X^{(q-1)+*}$ species which can be studied by photon spectroscopy.

In recent years there has been a significant progress in the study of low energy multiply charged ion-atom collisions. Total charge exchange cross-sections are now well established. A number of experiments have provided more detailed information about the collision dynamics. Measurements on $\mathrm{n} \ell \mathrm{m}$ population distributions requiring precise analysis using photon, electron and energy gain spectroscopy are in rapid development [8]. New theoretical tools for interpretation of such measurements have been also developed. Contribution of photon spectroscopy has been and remains very decisive.

In this paper, we rely on collisional results to examine the possibilities presented by new generation of multiply charged ion sources and more precisely ECRIS to study ion structures from the photon spectroscopy of species produced by electron capture in a gas target.

\section{2 - TOTAL ELECTRON CAPTURE CROSS-SECTIONS}

Extensive experimental data exist for electron capture in $\mathrm{H}, \mathrm{He}, \mathrm{H}_{2}$, Ar and $\mathrm{Kr}$ targets. Simple estimates based on the classical cross-sections introduced by Bohr and Lindhard [9] for multiply charged ions lead to good first approximations of the absolute magnitude of the cross-sections for any target atom.

For small ion velocity $v$, Knudsen et al. [10] found that $\sigma_{q, q-1}$ is proportional to $q$ and independent of $v$. Furthermore, for all but the lightest atoms, $\sigma_{q}, q-1 q^{-1} \propto Z^{2 / 3} I^{-1}$, where $Z$ and $I$ are the atomic number and ionisation potential of the target atom. This scaling $\sigma_{q,} q-1 q^{-1}$ agrees with that obtained by Ryufuku et Watanabe [11] from a distorted wave approximation for bare ions in atomic hydrogen target but is at variance with that found by Janev et Presnyakov [12] using the first Born approximation $\left(\sigma_{q, q-1} q^{-2}\right)$. Bliman et al. [13] suggested an experimental scaling law $\sigma_{q} . q-1$ $\simeq 4.10^{-12}\left(\mathrm{q} /\left[\mathrm{I}_{\mathrm{T}}(\mathrm{eV})\right]^{3}\right) \mathrm{cm}^{2}$ deduced from a series of their measurements. An overall agreement is 
found for the absolute magnitude of capture cross-section. The constant $\left(\sigma_{q \rightarrow q-1}\right) / q$ is very large and ranges from $2.10^{-16} \mathrm{~cm}^{2}$ to $2.10^{-15} \mathrm{~cm}^{2}$ depending on the target atoms. Higher values are obtained with higher $Z$ targets. Capture cross-section declines very rapidly at ion beam energies above some dozens of $\mathrm{keV} /$ nucleon.

For larger ion velocities the cross-section is proportional to $\mathrm{q}^{3}$ and declines very rapidly as $\mathrm{v}^{-7}$.

At LAGRIPPA, Grenoble, we used to work in the low energy range 2q-10q keV where electron capture cross-sections are large and somewhat constant.

Single electron capture is the only process available in a one-electron target [11]. In twoelectron targets $\left(\mathrm{H}_{2}, \mathrm{He}\right)$ double capture can occur. Some data for double capture cross-sections have been obtained. Generally they are 4 to 10 times smaller than the single capture cross-sections, depending on the collision parameters $[14,15]$. However, at sufficiently low energies, highly charge ions, when passed through helium or molecular hydrogen gas, should have a harger cross-section for two-electron loss than for one-electron loss [16]. In multi-electron targets (Ar, $\mathrm{Kr}, \ldots$ ) multielectron captures have been observed $[17,18]$, however with small cross-sections.

\section{3 - SPECTROSCOPY OF MULTIPLY CHARGED IONS}

Quasi-molecular descriptions [19] of slow collision, at velocity of active electrons larger than velocity of collision, show that the electrons are captured in excited states. Following their excitation in the target. most of the ions undergo spontaneous decay and the resulting photons can be analysed with various spectrometers adapted to the energy of emitted photons from $X$ and V.U.V. to visible ranges. This source of light is very efficient because of the selectivity of the excitation into a few excitud states. Its brightness depends essentially on the intensity of the incident ion beam.

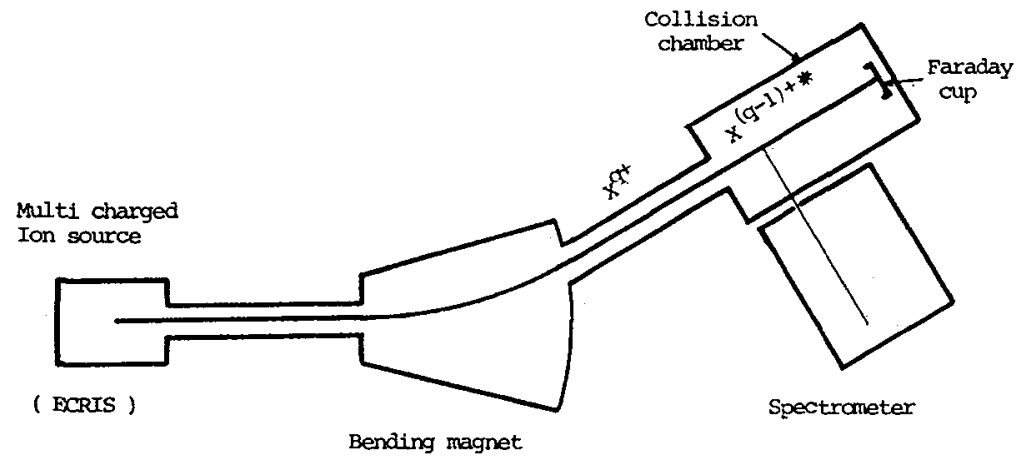

Fig.1 - Schematic arrangement of low energy beam-gas experiment.

\section{3-1 Experimental method. Line identification}

a) One-electron target. Spectra of one given charge state - When, after mass and charge analysis, an incident ion $\mathrm{X}^{\mathrm{q}+}$ undergoes a low energy charge exchange in an atomic hydrogen target, the only possible product is an excited $X^{(q-1)+*}$ ion. The excitation is strictly limited to one charge state. 
This is the method available to spectroscopists to observe light from excited states of one given charge state of a given isotope and to analyse its structure without being worried by blendings and extraneous contributions. The only constraint is to fulfill single collision conditions.

Such spectra. using an atomic hydrogen target, have been observed in a few highly ionised elements as lithium- and hydrogen-like $\mathrm{C}, \mathrm{N}$ and 0 [20]. They have been recorded in the only view to determine capture cross-sections. Experiments with atomic hydrogen are hard to perform because the production of a target with a high degree of dissociation and a high density is difficult. In most of atomic hydrogen targets the molecular component remains significant giving the possibility to the incident ion to capture two electrons and leading to a contamined spectrum.

Lithium vapor is also a good candidate for giving one charge spectra. Electron capture from $\mathrm{Li}$ at low impact energy involves the (2s) electron almost exclusively because of its small binding energy. Other electrons in inner shell are much more bound and no double electron capture has to be expected.

Potential applications are very interesting. First it is an help to assign the charge state to which an unidentified line must be attributed. More important maybe would be the use of this clean light source to measure very precisely wavelengths for Lamb-shift determinations in one-, two- and three-electron ions without the blending by $X$-ray satellites observed in beam-foil and recoil ion sources and with reduced Doppler effects.

Such experiments would need a well defined high intensity bare, one- or two-electron ion beams and a high density purely atomic hydrogen target. However satellite lines due to double capture in molecular hydrogen impurity could be corrected by comparing spectra obtained in the same ion beam passing through and atomic and a molecular hydrogen targets.

In consideration of the difficulty to use an atomic hydrogen target, and to avoid complications introduced by multi-electron targets, most of the spectroscopic results have been and will be obtained using two-electron targets, namely helium and molecular hydrogen. This light source possesses a number of attractive features that we will review now.

b) High chemical and isotopic purity of che light source - The momentum analysis in a magnetic field of the accelerated ions should in principle guarantee a chemically and even isotopically pure beam through the exciter. Contaminations in the beam can occur from species having the same $\mathrm{q} / \mathrm{m}$. A simple case is given by $\mathrm{q} / \mathrm{m}=1 / 2$ as, e.g., $14 \mathrm{~N}^{7+}$ and $160^{8+}$ nuclei. The mixing can be avoided by using different isotopes as, e.g., $15 \mathrm{~N}^{7+}$.

Contaminations from the gas target can be studied by comparing spectra obtained using different gases and, more simply, by using pure gases presenting well known emission spectra as $\mathrm{H}_{2}$ and $\mathrm{He}$. Target lines can also be recognized from their Doppler modifications. The Doppler width is narrower for the target atoms and when the light is observed at an angle from the ion beam axis, the beam lines are Doppler shifted relative to the target lines. Furthermore the target atoms are only weakly excited. This kind of contamination is not a problem at all. 
c) Charge of ions emitting spectral lines - An ion of incident charge q emerges with a charge q-1 or q-2 after charge transfer from a two-electron target as He or $\mathrm{H}_{2}$. Generally the lines emitted by ions of charge q- 1 are much stronger than the lines due to ions with charge $q-2$.

By varying the energy it is possible to change the ionisation states produced by the collision. Observed lines to be assigned to charge state $q-2$ would be strengthered at lower energy.

of more precise application is the comparison of spectra obtained successively using incident beam charges $q$ and $q-1$, as demonstrated by the figure 2 . Lines which are observed in both spectra can be assigned to charge state $\mathbf{q}-2$.



Fig.2-Spectra obtained during (a) $\mathrm{Ar}^{8+}+\mathrm{He}$ and (b) $\mathrm{Ar}^{7+}+\mathrm{He}$ collisions.

Peaks marked with stripes, common to both spectra, are thus due to transitions in Ar VII.

This property of the low energy charge exchange light source to produce lines which can be assigned without any ambiguity to a given charge is unique. It is a great help for the identification of transitions which have not been reported earlier. Techniques for attributing a charge state to an observed line exist also in beam-foil method but they are more difficult to apply and are generally less selective. Recoils ions are produced in many charge states by a broad band excitation process. 
d) Total light inceasity - Photon spectroscopy can achieve a very high resolution. Its main disadvantage is in its extremely low detection efficiency due to the acuteness of the acceptance solid angle of monochromators and to the low quantum efficiency for photon detection, specially in some wavelength ranges. The brightness of the light source has to be sufficient to achieve the requested spectral resolution.

We have noted in the section 2 above that capture cross-sections grow rapidly with charge state of incident ions and depend on the nature of the target gas. They are greater for $\mathrm{H}_{2}$ and $\mathrm{H}$ than for He (Fig.3). More generally the capture cross-section grows as the ionisation potential of target gas reduces. Models also predict that capture into the ground state should be negligible and that emergent ions, after charge exchange, are produced in highly excited states. The combination of high total capture cross-sections by choosing the right conditions for velocity and target and selectivity of excited levels production result in a sufficient intensity of lines to be spectroscopically analysed.

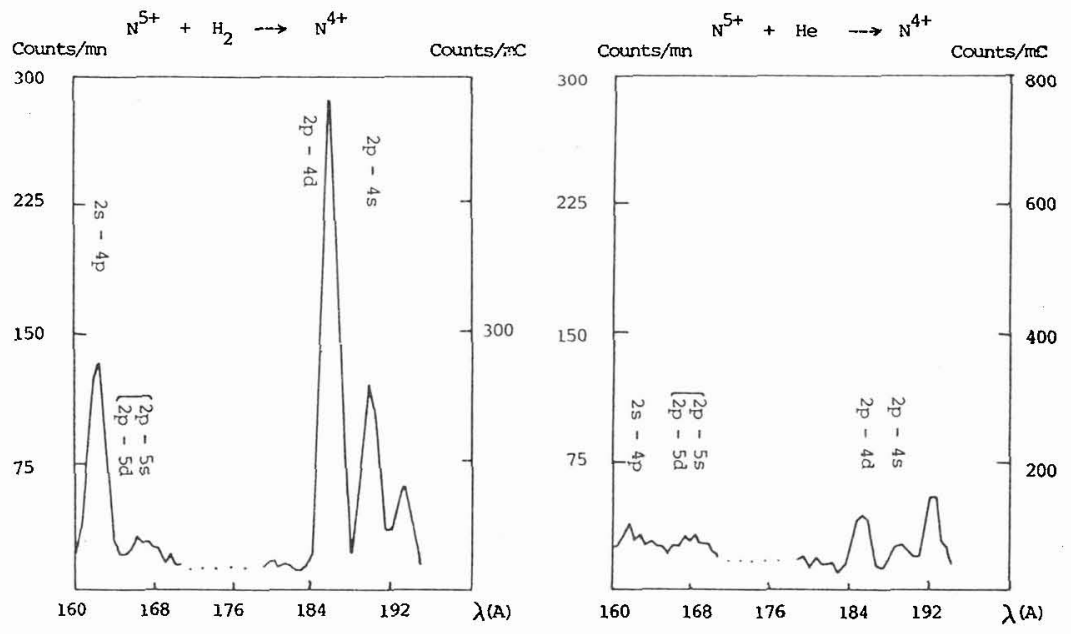

Fig. 3 - Comparison of intensities in $\mathrm{N}^{5+}+\mathrm{H}_{2}$ and $\mathrm{N}^{5+}+$ He collisions for a given number of incident particles. $\mathrm{H}_{2}$ is more efficient to excite $\mathrm{N} V$ spectrum than $\mathrm{He}$.

e) Shell and substell excitation - In what electronic state is the ion formed ? Some extended calculations and several approximate models of low energy charge exchange process have been developed [12]. From theoretical approches ones may predict in some extend what $\boldsymbol{n}$ is prominently populated. The observed lines have to be assigned to transitions from upper levels $\mathbf{n}_{\text {sup }} \leq$ maximum populated $n$. If transition probabilities are known or can be easily calculated, the most probable transitions to be observed in beam-gas spectra can be deduced. In the classical barrier model [21] $n$ increases as $q^{3 / 4} / \sqrt{I_{B}}$. This model can be used to predict the dominantly populated $n$ shell. Its success has been demonstrated by many of published results.

For a given target gas higher charged ions capture electrons in higher $n$ principal quantum numbers. 
For a given charge $q$, a high ionisation potential target has to be chosen to obtain capture in low n state giving rise to simple spectra containing only a few transitions from low lying levels. By changing to lower ionisation potential targets, successive higher $n$ states are populated, and new transitions arise completing the preceding spectra (Fig.4). Rydberg levels are excited when using low ionisation potential alkaline atoms as targets. Examples will be discussed in the following section 3.2d).
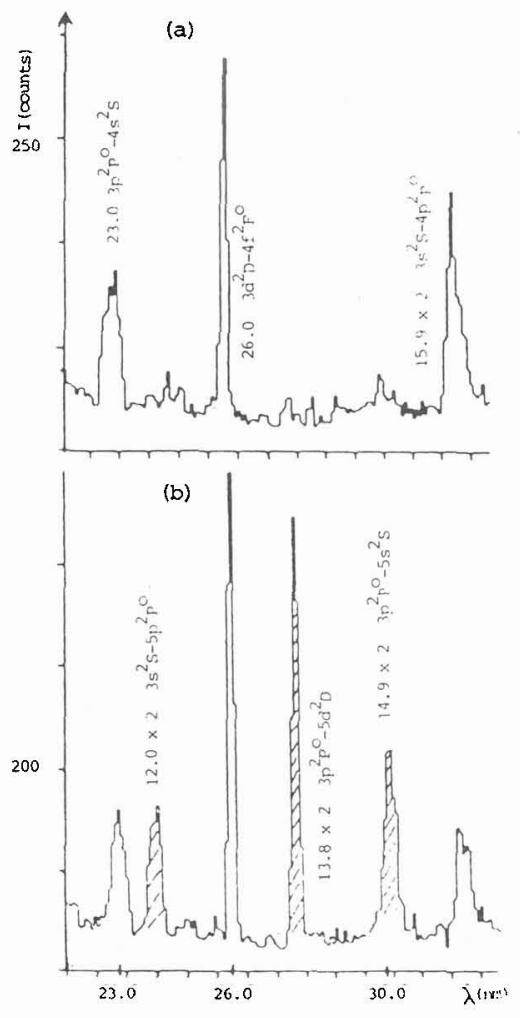

Fig.4 - Comparison of spectra obtained during the collisions $\mathrm{Ar}^{8+}+\mathrm{He}(\mathrm{a})$ and $\mathrm{Ar} \mathrm{r}^{8+}+\mathrm{H}_{2}$ (b). Peaks marked with stripes, appearing only with $\mathrm{H}_{2}$, are issued from $n \geq 5$.

The assignment of lines to a given subshell from collision conditions is much more difficult. The distribution over $\ell$ subshells within each $n$ shell are energy dependent, generally shifting to larger $\ell$ values with increasing energy. At high energy a statistical ordering is expected.

1) Spin conservation. Assigament of observed lines to a given multiplicity - Systems of different multiplicity are generally excited. For example, heliumlike ions contain both triplet and singlet systems. The contribution of the singlet terms is estimated at one third of the triplet. When contributions are resolved this is generally verified. The relative intensity of observed lines leads to their attribution to one system or the other. 
However the Wigner spin conservation rule requires that the total electron spin angular momentum of pair of atoms does not change in course of a collision. A simple case is given by the double capture from helium or noble gas $(s=0)$ by heliumlike ions $(s=0)$. The spin of the produced berylliumlike ion is then zero. In this case the transitions to assign observed lines are only singlets. No triplet lines are possible. As an illustration, part of spectrum [22] obtained in collision $\mathrm{N}^{5+-H e}$ at $50 \mathrm{keV}$ and displayed in Fig. 5 shows that strong lines are due to single electron capture though weak lines are assigned to transitions between singlet terms of N IV produced by double capture.

Spin conservation has been found valid for light target $[22,23]$ and not for heavier targets and monocharged incident beams [24].



Fig.5 - N IV spectrum obtained by double electron capture. Peaks marked by a black point are issued from $2 \ell 3 \ell^{1} 2 \mathrm{~L}$ terms of $\mathrm{N}^{3+}$.

g) Core conservation and core change. Doubly excited transitions - Observed excited ions are generally produced by single capture of an excited electron by the incident ion in its ground state. However the probability that the electron capture to be accompanied by excitation of an electron of the incident particle is not entirely negligible. Most of the observed lines can be assigned to transitions between singly excited states.

In comparison with core changing capture, the relative importance of core conserving capture rises with $v$, maybe because the electron capture with additional rearrangment consumes more time. At intermediate velocity the spectra would be simpler than at lower velocity excluding most of the doubly excited states.

h) A technique to enhance the production of doubly excited states - To en hance the production of doubly excited states we have proposed and realised a new technique. $A \mathrm{X}^{(q+1)+}$ ion beam issued from 
a highly charged ion source is sent through a first gas cell. The emergent beam component $\mathrm{X}^{\mathrm{q}^{+}}$is charge analysed. It may contain a jarge fraction of metastable ions in an helium or neonlike ion beam. Pressure in the target gas has been ajusted to produce more than $50 \%$ metastable ions. This $\mathrm{X}^{\mathrm{q}^{+}}$beam is sent through a second gaseous cell for electron capture $\mathrm{q} \rightarrow(\mathrm{q}-1)$. The fraction $\mathrm{X}^{\left(\mathrm{q}^{+}\right)^{*}}$ of the beam which is in a metastable state capture an electron to give doubly excited $\mathbf{X}^{(q-1)^{* *}}$ ions. Illustration of the use of this new technique for a very efficient production of doubly excited atoms will be given later in section $3.2 \mathrm{c}$ ).

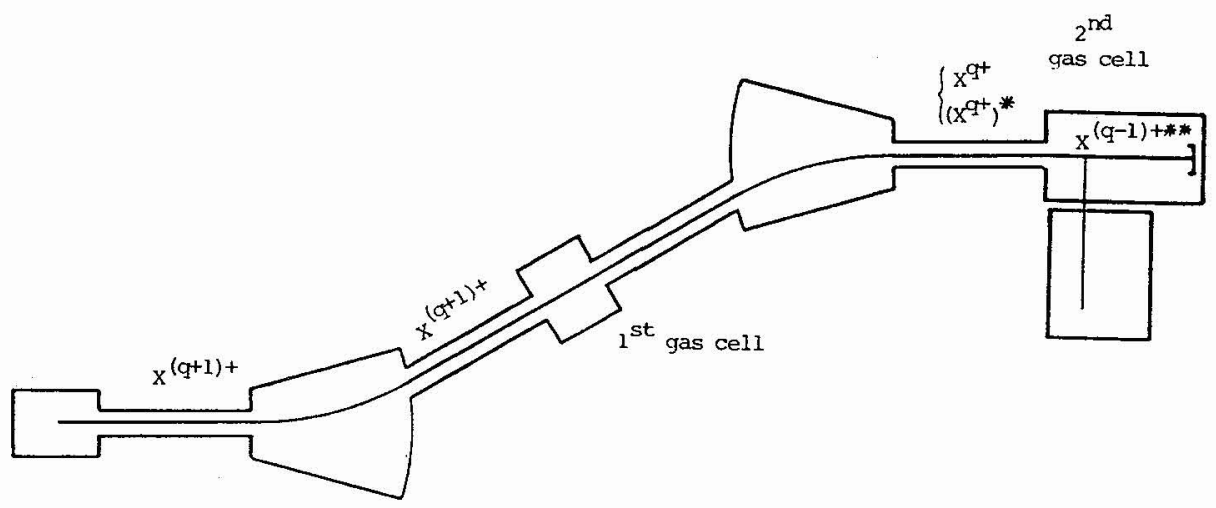

Fig.6 - Schematic arrangement of a low energy beam-gas experiment using an ion beam "prepared" in a metastable state.

\section{3- 2 Spectroscopic results}

Spectroscopic results as to wavelengths and energy levels obtained by this method are till now passably scarce. Experiments with low energy highly ionised atoms have been aimed to measurements of intensities rather than wavelengths. A very few spectra have been recorded with a sufficient resolution to be useful for structure analysis. On the side results originating from beamgas experiments have provided interesting spectral informations on highly ionised aluminium [24], titanium [[25], argon [26,27] and krypton $[26,27]$.

New spectral materials we have obtained from beam-gas interaction at LAGRIPPA are presented in two categories. the first one includes new informations for atomic ions which have been incompletely studied by other techniques. The second category includes results on doubly excited states which can be abundantly populated by the beam-gas light source, specially using our two cell method.

a) Completion of previously incompletely studied systems - A low resolution spectroscopic study of Al VIII has been undertaken by Mayo et al. [24]. The complexity of the spectrum, the lack of information on the possibility of high $n$ shell to be or not excited have lead to an number of misidentifications in the list of suggested transitions. This spectrum would be recorded at better resolution to give precise spectroscopic information. 
Our spectrum of $\mathrm{Ti} X I I I$ [25] has been a demonstration of the feasability and reliability of the beam-gas method for the study of a complex spectrum with a resolution comparable to that obtained with usual beam-foil technique. Results were just a confirmation that a previous attribution of beam-foil lines to Ti XIII $\left(3 \ell-3 \ell^{\prime}\right)$ was correct. We have also detected a line 5-6 from a n=6 level which is eventually populated by beam-gas with a good efficiency.

However most of the new data on singly excited states which originate from beam-gas experiments concern Ar VII, Ar VIII, $\mathrm{Kr}$ VII, $\mathrm{K}$ VIII, $\mathrm{Kr}$ IX.

Low wavelength lines have generally been measured at several orders to get more resolution and to confirm identifications. Some of the lines we observed in Ar VII are due to decays from displaced terms : they will be discussed in the next section. Most of the observed lines decay from singly excited states $1 s^{2} 2 s^{2} 2 p^{6} 3 s n \ell$. Some, previously observed by other authors, have been wavelength corrected ( $3 s$ is and $\left.5 s^{1} p \rightarrow 3 s 3 p^{1} p^{0}\right)$. Others have been observed for the first time ( $3 s$ sp and $4 p$ $3 \mathrm{p}^{0} \rightarrow 3 \mathrm{~s} 4 \mathrm{~s}^{3} \mathrm{~s}$ and $3 \mathrm{~s} 3 \mathrm{~d}^{3} \mathrm{D}$ ). Some new lines, observed in Ar VIII between $42 \mathrm{~nm}$ and $74 \mathrm{~nm}$, have been attributed to transitions 5-4. Assignment of unknown lines to Ar VII or Ar VIII have been determined from the comparison of spectra obtained using $\mathrm{Ar}^{7+}$ and $\mathrm{Ar} \mathrm{r}^{8+}$ incident ions successively. Assignment to a given $n$ value for upper levels of transition has been helped by comparison of spectra obtained successively in $\mathrm{He}$ and $\mathrm{H}_{2}$ targets. Work is going on analysing spectra of Ar VI with the hope of contributing to unambigous identifications of lines observed by beam-foil and by recoil ion spectroscopy which have received divergent assignments.

Some techniques of identification have been applied for $\mathrm{Kr}$ VII and $\mathrm{Kr}$ VIII. A large number of energy levels of $\mathrm{Kr}$ VII are unknown. Only the $\mathrm{n}=4$ levels have been tabulated. As a consequence the analyse of our Kr VII spectra has been made difficult. From fourteen new lines tentatively assigned to $4-5$ transitions we were able to determine five new levels $\left(5 s, 1,3 \mathrm{~S}, 5 \mathrm{p}, 1 \mathrm{P}\right.$ and $3 \mathrm{P}_{0,1,2,5 \mathrm{~d}}$ 3D). In $\mathrm{Kr}$ VIII we have assigned six new lines to 6-4 and 6-5 transitions using the theoretical data of Cheng and $\mathrm{Kim}$ [28] and Lindgard et al. [29]. Agreement with theory is generally good inside the error bars. However there are significative shifts between our experimental results and the data of Cheng et $\mathrm{Kim}$ for the $6 s_{1 / 2}, 6 \mathrm{~d}_{3 / 2.5 / 2}$ and $6 \mathrm{f}_{5 / 2,7 / 2}$ levels.

All the $\mathrm{Kr}$ IX lines emitted during the collision of $\mathrm{Kr}^{9+}$ with $\mathrm{H}_{2}$ at $1 \mathrm{keV} / \mathrm{a} . \mathrm{m} . \mathrm{u}$. between 40 and $70 \mathrm{~nm}$ were observed for the first time [26]. They have been tentatively identified to transitions from $n=4$ levels following calculations of Wyart and Ryabtsev 130 ].

b) Displaced terms - In spectra of berylliumlike Ne VII, obtained from an incident beam of lithiumlike ground state $\mathrm{Ne}^{7+}$ ions, some weak lines are present which are not issued from normal excited terms $1 s^{2} 2 s \mathrm{n} \ell^{1,3} \mathrm{~L}$ produced by core conserving electron capture (Fig.7). They have been identified as known transitions from displaced terms $1 \mathrm{~s}^{2} 2 \mathrm{p} n \ell 1,3 \mathrm{~L}$ which converge to the first excited term 2p $2 \mathrm{P}$ in Ne VIII [31]. This indication that charge exchange is able to populate such levels has been for us an incitation to look at such transitions in higher $Z$ ions and in other electronic sequences. In magnesiumlike Ar VII, some lines which are only present in collision on He target are due to transitions from displaced terms $1 s^{2} 2 s^{2} 2 p^{6} 3 p n \ell$. We have tentatively assigned observed new lines to transitions from $3 p d^{1} F^{0}, 3 p 4 p{ }^{1} D, 3 p$ $4 p^{3} S$. 


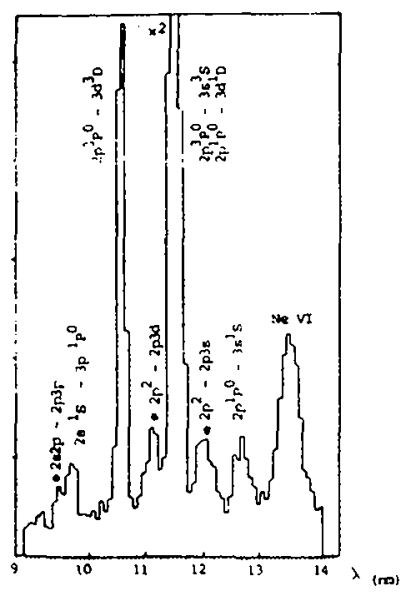

Fig. 7 - Ne VII spectrum transitions between displaced $1 s^{2} 2 \mathrm{p} n \ell{ }^{3}$ L terms of NeVII are marked with an asterisk.

c) Doubly excited systems - A doubly excited state of an ion has two electrons excited relative to the ground state. Doubly excited states of $L i$ I formed from $1 \mathrm{~s} 2 \mathrm{~s} \ell$ and $1 \mathrm{~s} 2 \mathrm{p} \mathrm{n} \ell$ are $50-70 \mathrm{eV}$ above the ground state and this largely above the ionisation potential. However quartet states are metastable against autoionisation to the normal $\mathrm{Li} I$ doublet continuum. Such states are abundantly populated by beam-foil interaction. They were observed from optical transitions by Buchet et al. [32] and Bickel et al. [33] twenty years ago. Since then this quartet system has been studied in detail [34] in heavier ions of the sequence up to Si XII 1351.

Beam-gas spectroscopy is the ather possibility for efficiency producing such states, using the experimental method described above (section $3.1 \mathrm{~h}$ )). Our first experiment has been with $\mathrm{CIV}^{* *}$. A more detailed analysis has been performed on $\mathrm{N} \mathrm{V}^{* *}$. A $\mathrm{N}^{6+}$ ion beam produced by the Grenoble ECR ion source and selected by a first magnet, is sent through a gas target. The $\mathrm{N}^{5+}$ ions produced here by electron capture and selected by a second magnet are sent through a second gas target $\left(\mathrm{H}_{2}\right)$. The $\mathrm{N} V$ spectrum then observed is very different of that produced by electron capture of $\mathrm{N}^{5+}$ delivered by the ECR ion source itself from the same gas target (Fig.8). By substracting the "direct" spectrum from the "prepared" one, the series of lines due to transitions between doubly excited states are clearly obtained. This is an appreciable advantage of beam-gas method compared to beam-foil technique. Many lines have been identified. From two new lines the energy of $1 s^{2} 2 s$ ap ${ }^{40}$ state has been determined. Other lines observed for the first time complete the diagram of $N^{* *}[36]$.

d) Doubly excited Rydbers stales of As VIII, with $a=7, \ldots, 12 ; 8-6, \ldots, 11$ aad $j=17 / 2, \ldots, 27 / 2$ - Compared to $\mathrm{Li}$ I sequence, in the heavier alkali sequences $\mathrm{LS}$ coupling breaks down, producing strong quartet doublet mixing and rapid autoionisation. However in the $\mathrm{Na}$ sequence states of the highest $\mathrm{L}$ and $\mathrm{J}$ of a given multiplet can be optically observed as they will not mix strongly. The transition $3 s$ sp ${ }^{4} \mathrm{D}_{7 / 2}$ 3s 3d ${ }^{4} \mathrm{~F}_{9 / 2}$ has been tentatively indentified in Na I by Berry et al. 137] and in Mg II by Lundin el al. [38]. More recently the $\mathrm{Na} \mathrm{I}^{* *}$ spectrum has been revisited by Holmgren et al. 139].

We have observed $\Delta \mathrm{n}=1$ and $\Delta \mathrm{n}-2$ transitions from $2 \mathrm{p}^{5} 3 \mathrm{~s} 7 \mathrm{i}, 8 \mathrm{k}, 9 \ell$ and $11 \mathrm{~m}$ configuration in the 
quartet spectra of Nalike Ar VIII after double collisions between low energy Ar ${ }^{9+}$ ions and cesium vapor. Strong lines obtained in the visible range by one electron capture by $\mathrm{Ar}^{8+}$ and $A \mathrm{r}^{9+}$ beams at low pressure of cesium are due to $\Delta n=1$ and $\Delta=2$ transitions from upper levels $n=12,11, \ldots, 8$ in consistence with classical barrier models. After $\mathrm{Ar}^{9+}-\mathrm{Cs}$ collisions, one observe, at high pressure and good spectral resolution, in addition to strong Ar IX Rydberg lines, weak Ar VIII Rydberg lines between $1 s^{2} 2 s^{2} 2 p^{6}{ }^{4} \mathrm{~L}$ terms and satellite lines due to transitions between $1 s^{2} 2 s^{2} 2 p^{5} 3 s n l^{4} L$ terms which can be produced from the metastable $1 s^{2} 2 s^{2} 2 p^{5} 3 s \mathrm{P}_{0.2}$ terms of neonlike Ar IX. The satellites which are optically observed are those with higher $J$ as, for example, $1 s^{2} 2 s^{2} 2 p^{5} 3 s$ i ${ }^{4} K_{17 / 2}-1 s^{2} 2 s^{2} 2 p^{5} 3 s 8 k^{4} L_{19 / 2}$ or $3 s 9 \ell^{4} M_{21 / 2}-3 s 11 m{ }^{4} N_{23 / 2}$ (Fig.9).



Fig. 8 - Spectra of $\mathrm{N} \mathrm{V}$ during the collision of (a) Incident $\mathrm{N}^{5+}$ beam from ECRIS (mainly in ground state). (b) Incident $\mathrm{N}^{5+}$ beams prepared by $\mathrm{N}^{6+}+\mathrm{H}_{2}$ charge exchange (rich in metastable $\mathrm{N}^{5+1}$ (s $2 \mathrm{~s}^{3} \mathrm{~S}$ ).

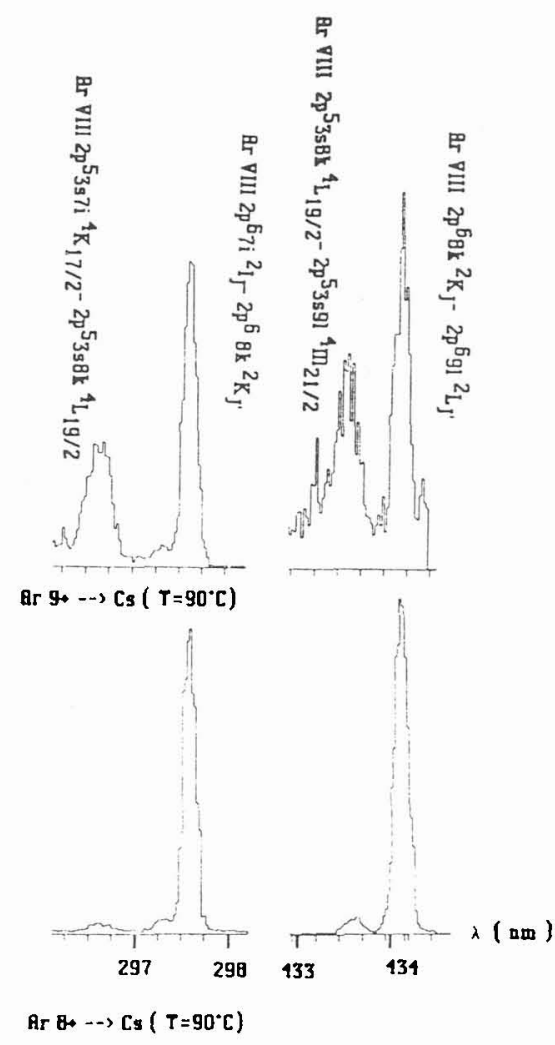

Fig.9 - Transitions $8 \mathrm{k}-9 \ell$ and $7 \mathrm{i}-8 \mathrm{k}$ in Ar VIII. (a) Ar VIII $2 p^{6} 8 k^{2} K_{J}-2 p^{6} 92^{2} L_{J}$ and $2 p^{6} 7 i$ ${ }^{2} C_{J}-2 p^{6} 8 k^{2} K_{J}$, following the collision $A r^{8+}$ Cs. (b) Ar VIII $2 p^{5} 3 s 8 k^{4} L_{19 / 2}-2 p^{5} 3 s 98$ ${ }^{4} M_{21 / 2}$ and $2 p^{5} 3 s i^{4} K_{17 / 2}-2 p^{5} 3 s$ sk ${ }^{4} L_{19 / 2}$ following the double collision $\mathrm{Ar}^{9+}-$ Cs. 


\section{4 - CONCLUSIONS}

Low energy ion beams excited by electron capture from gases have several advantages over conventional spectroscopic sources.

- The beam-gas source can be made isotopically pure, just as the beam-foil is.

- Selected high charge states can be obtained. However, if low energy beam-gas collisions are more selective than beam-foil interaction, highest charge states are not yet available at existing ion sources. One charge state is obtained in a single electron, target gas. In multi-electron gases, the charge state can be surely assigned by comparing lines in spectra obtained using successive incident charge states. That makes a great difference with recoil ion light source in which all charge stațes are present.

- Selected $\mathrm{n} \ell$ subshells can be excited. A few $n$ shells are populated, the number of which decreases with decreasing ion velocity. Higher $n$ shells are obtained when using low ionisation potential target gas.

- Spin conservation is obeyed in low 2 target. This can lead, in some cases, to simplification in the spectra, only levels of a given multiplicity being populated.

- Doubly excited states are populated, mainly at low velocity. They can be selectively excited by using two successive collisions. By choosing a low ionisation potential target, doubly excited Rydberg levels can be populated.

- States appear to have in many cases an aligned sublevels population.

- Doppler effects are present as in all techniques using ion beams. They are less important than in beam-foil experiments on ions of same charge states and they have not been a limitation for spectra as yet studied.

Already in very first years of study of low energy charge exchange processes with highly charged ions, a number of exciting and challenging results have been obtained. These works include measurements of previously un reported wavelengths in uncompletely known normal systems, displaced terms, low lying doubly excited states, and doubly excited Rydberg. We know now that low energy beam-gas experiment will be used as a spectroscpic light source presenting outstanding properties. Future warks will need higher spectral resolution and will lead to new types of experiments.

\section{REFERENCES}

[1] H.D. Betz, Rev. Mod. Phys. 44 (1972) 466.

[2] S. Martin, J.P. Buchet, M.C. Buchet-Poulizac, A. Denis, J. Desesquelles, J.P. Grandin, D. Hennecart, X. Husson. D. Lecler, I. Lesteven, Phys. Rev. A 35 (1987) 2327 ;

J. Desesquelles, Nucl. Instr. and Meth. in Phys. Res. B 31 (1988) 30.

[3] H. Gould, D. Greiner, P. Lindstrom, T.J.M. Symons, H. Crawford, Phys. Rev lett. 52 (1984) 180.

14! D.A. Church. S.D. Krairs. M. Merou. B.M. Johnson. K.W. Jones. I.A. Sellin, L.S. O. J.C. Levin. R.T. Short. Nucl. Instr, and Meth. in Phys. Rev. B 31 (1988) 262.

[5] G.S.I. Report. 
[6] D.J. Nagel in "Beam-Foil Spectroscopy", ed. by I.A. Sellin and D.J. Pegg(Plenum, 1976), p. 961.

[7] R. Geller, B. Jacquot, Nucl. Instr. and Meth. 184 (1981) 293

[8] Proceedings of this conference.

[9] N. Bohr, J. Lindhard, K. Dan, Vidensk Selsk. Mat. Fys. Medd. 28 (1954) 407.

[10] H. Knudsen, M.K. Haugen, P. Hvelplund, Phys. Rev. A23 (1981) 597.

[11] H. Ryufuku. T. Watanabe, Phys. Rev. A20 (1979) 1828.

[12] R.K. Janev, L.R. Presnyakov, Phys. Report 70 (1981) 1.

[13] S. Bliman, M. Bonnefoy, J.J. Bonnet, S. Dousson, A. Fleury, D. Hitz, B. Jacquot, Physica Scr. T3 (1983) 63.

[14] R.K. Janev, L.P. Presnyakov, V.P. Shevelko, in "Physics of Highly Charged Ions", ed. by G. Ecker (Springer, 1985), $n^{\bullet} 13$.

[15] D.H. Crandall, M.L. Mallory, D.C. Kocher, Phys. Rev. A23 (1981) 597

[16] D.H. Crandall, R.E. Olson, E.J. Shipsey, J.C. Browne, Phys. Rev. Lett. 36 (1976) 858.

[17] H. Klinger, A. Muller, E. Salzborn, J. Phys. B8 (1975) 230.

[18] S. Bliman, S. Dousson, R. Geller, B. Jacquot, D. Van Houtte, Physica Scr. 23 (1981) 184.

[19] M. Barat, V. Sidis, Ann. Phys. Fr. 8 (1983) 133.

[20] D. Dijkkamp, D. Ciric, E. Vlieg, A. de Boer, F. J. de Heer, J. Phys. B 18 (1985) 4763.

[21] H. Ryufuku, K. Sasaki, T. Watanabe, Phys. Rev. A 21 (1980) 745.

[22] P.H. Cotte, Thesis, Lyon (1984):

P.H. Cotte, M. Druetta, S. Martin, A. Denis, J. Désesquelles, D. Hitz, S. Dousson, Nucl. Instr. and Meth. in Phys. Rev. B9 (1985) 743.

[23] H. Cederqvist, L.H. Andersen, A. Barany, P. Hvelplund, H. Kundsen, E.H. Nielsen, J.O.K. Pedersen. J. Sorensen, J. Phys. B 18 (1985) 3951.

[24] M. Mayo, D. Hitz. M. Druetta, S. Dousson, J.P. Desclaux, S. Bliman, Phys. Rev. Lett. 54 (1985) 317.

[25] S. Martin, M. Druetta, J. Desesquelles, Nucl. Instr. and Meth. in Phys. Rev. B 14 (1986) 254.

[26] M. Druetta, T. Bouchama, S. Martin, J. Désesquelles, J. Physique 49 C-1 (1988) 365.

[27) T. Bouchama. Thesis, Lyon (1988).

[28] K.T. Cheng, Y.K. Kim. At. Data and Nucl. Data Tables 22 (1978) 547.

[29] A. Lindgard, L.J. Curtis, I. Martinson, S.E. Nielsen, Physica Scr. 21 (1980) 47.

[30] J.F. Wyart, A.N. Ryabtsev, Physica Scr. 33 (1986) 215.

(31) M. Druetta. T. Bouchama, S. Martin, J. Phys. B 19 (1986) L-723.

[32] J.P. Buchet, A. Denis, J. Désesquelles, M. Dufay. Phys. Lett. 28 A (1969) 529.

[33] W. Bicke1, I. Bergstróm, R. Buchta, L. Lundin, I. Martinson, Phys. Rev. 178 (1969) 118.

[34] K.G. Berry, Physica Scr. 12 (1975) 5.

[35] J.P. Buchet, M.C. Buchet -Poulizac, A. Denis, J. Desesquelles, M. Druetta, S. Martin, Y. Ouerdane, J. Physique, 49. C-1 (1988) 111.

136] T. Bouçhama, A. Denis, J. Désesquelles, M. Druetta, M. Farizon, S. Martin, Nucl. Instr. and Meth. in Phys. Rev. B 31 (1988) 367

[37] H.G. Berry, R. Hallin, R. Sjodin, M. Gaillard, Phys. Lett. 50A (1974) 191.

[38] L. Lundin, B. Engman, J. Hilke. I. Martinson, Physica Scr. 8 (1973) 274.

[39] D.E. Holmgren, D.J. Wolker, D.A. King. S.E. Harris, Phys. Rev. A 31 (1985) 677. 